\title{
Total Parenteral Nutrition in Patients Following Pancreaticoduodenectomy: Lessons from 1184 Patients
}

\author{
Cullen Worsh \\ Thomas Jefferson University \\ Talar Tatarian, MD, PGY-4 \\ Department of Surgery, Thomas Jefferson University
}

Awinder Singh

Thomas Jefferson University

Michael J. Pucci, MD

Thomas Jefferson University

Jordan M Winter, MD

Thomas Jefferson University

Follow this and additional works at: https://jdc.jefferson.edu/surgeryposters

əPraxtodabe Eorrgediditiamahaortsors

Let us know how access to this document benefits you

\section{Recommended Citation \\ Worsh, Cullen; Tatarian, MD, PGY-4, Talar; Singh, Awinder; Pucci, MD, Michael J.; Winter, MD, Jordan M; Yeo, MD, Charles J.; and Lavu, MD, Harish, "Total Parenteral Nutrition in Patients Following Pancreaticoduodenectomy: Lessons from 1184 Patients" (2017). Department of Surgery Posters. 7. https://jdc.jefferson.edu/surgeryposters/7}

This Article is brought to you for free and open access by the Jefferson Digital Commons. The Jefferson Digital Commons is a service of Thomas Jefferson University's Center for Teaching and Learning (CTL). The Commons is a showcase for Jefferson books and journals, peer-reviewed scholarly publications, unique historical collections from the University archives, and teaching tools. The Jefferson Digital Commons allows researchers and interested readers anywhere in the world to learn about and keep up to date with Jefferson scholarship. This article has been accepted for inclusion in Department of Surgery Posters by an authorized administrator of the Jefferson Digital Commons. For more information, please contact: JeffersonDigitalCommons@jefferson.edu. 


\section{Authors}

Cullen Worsh; Talar Tatarian, MD, PGY-4; Awinder Singh; Michael J. Pucci, MD; Jordan M Winter, MD; Charles J. Yeo, MD; and Harish Lavu, MD 
BACKGROUND

$>$ Total parenteral nutrition (TPN) has historically been used conservatively in the management of patients after pancreaticoduodenectomy (PD).

$>$ In this study, we evaluate the indications for and outcomes associated with TPN use in a high-volume pancreatic surgery center.

\section{METHODS}

$>$ Following IRB approval, all cases of PD from December 2006 through December 2015 were reviewed utilizing our pancreatic surgery database.

$>$ Following identification of peri-operative TPN use, patients were separated into two cohorts with the hypothesis that was those patients who were started on TPN but received it for less than 3 days due to an improvement in their clinical condition, most likely were overprescribed.

Figure 1. Study Design

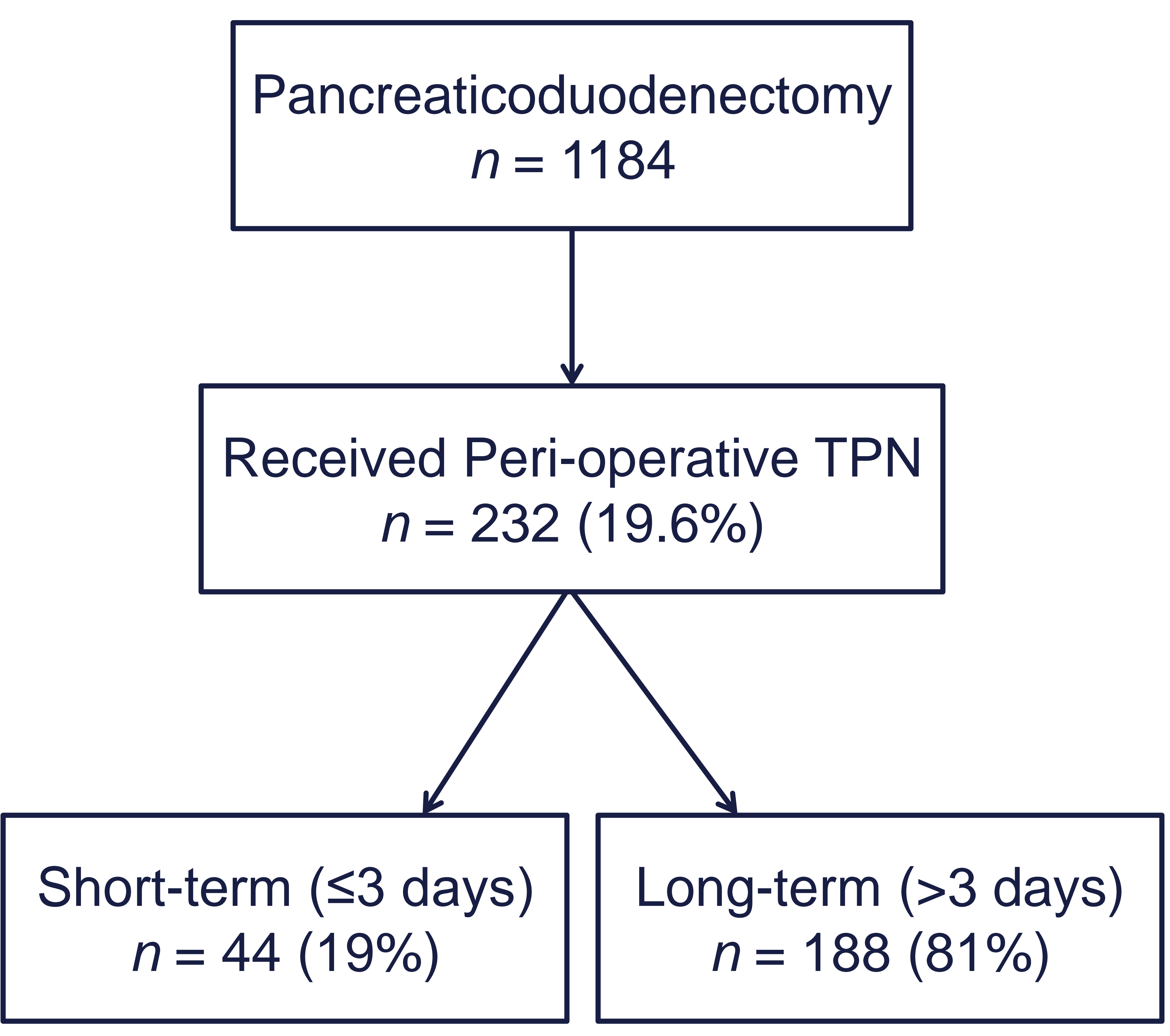

\section{RESULTS}

Table 1. Patient Demographics and Operative Data

\begin{tabular}{lccc} 
& No TPN & TPN & \\
& $n=952(80 \%)$ & $n=232(20 \%)$ & $p$-value \\
\hline Male & $453(48)$ & $156(67)$ & $\mathbf{0 . 0 0 0 1}$ \\
Age & $66.8(18-92)$ & $69.3(37-90)$ & $\mathbf{0 . 0 0 7}$ \\
BMI & $25.6(10.8-52.6)$ & $26(15-43.9)$ & 0.30 \\
Diabetic & $245(26)$ & $60(26)$ & 1.00 \\
Smoker (Current or Past) & $448(47)$ & $131(56)$ & $\mathbf{0 . 0 1}$ \\
Pylorus Preservation & $801(84)$ & $183(79)$ & 0.06 \\
EBL (mL) & $430(50-1600)$ & $630(40-8000)$ & 0.08 \\
Soft Gland (n=872;n=204) & $421(48)$ & $114(56)$ & $\mathbf{0 . 0 5}$ \\
Pathology & & & \\
$\quad$ PDA & $601(63)$ & $164(71)$ & $\mathbf{0 . 0 3}$ \\
IPMN & $138(15)$ & $27(12)$ & 0.29 \\
$\quad$ Pancreatitis & $52(5)$ & $6(3)$ & 0.09 \\
\hline
\end{tabular}

Table 2. Indications for and Patterns of TPN Use

\begin{tabular}{lccc}
\hline \multicolumn{1}{c}{ Complication } & $\begin{array}{c}\text { TPN } \leq 3 \text { Days } \\
n=44(19 \%)\end{array}$ & $\begin{array}{c}\text { TPN }>3 \text { Days } \\
n=188(81 \%)\end{array}$ & $p$-value \\
\hline Any Complication & $39(89)$ & $179(95)$ & 0.15 \\
DGE & $32(73)$ & $139(74)$ & 0.85 \\
Grade A & $25(78)$ & $59(42)$ & $\mathbf{0 . 0 0 0 3}$ \\
Grade B & $6(19)$ & $53(38)$ & $\mathbf{0 . 0 4}$ \\
Grade C & $1(3)$ & $27(19)$ & $\mathbf{0 . 0 3}$ \\
Pancreatic Fistula & $8(18)$ & $94(50)$ & $\mathbf{0 . 0 0 0 1}$ \\
Grade A & $5(63)$ & $24(26)$ & $\mathbf{0 . 0 4}$ \\
Grade B & $2(25)$ & $57(61)$ & 0.07 \\
Grade C & $1(13)$ & $13(14)$ & 1.00 \\
Deep Surgical Site Infection & $7(16)$ & $91(48)$ & $\mathbf{0 . 0 0 0 1}$ \\
Pneumoniae & $0(0)$ & $31(16)$ & $\mathbf{0 . 0 0 1}$ \\
\hline
\end{tabular}

Table 3. Postoperative Imaging

\begin{tabular}{lccc}
\hline Patient Imaging & $\begin{array}{c}\mathrm{TPN} \leq 3 \text { Days } \\
\mathrm{n}=44(\%)\end{array}$ & $\begin{array}{c}\mathrm{TPN}>3 \text { Days } \\
\mathrm{n}=188(\%)\end{array}$ & $\mathrm{p}$-value \\
\hline Upper GI Imaging & $20(46)$ & $109(58)$ & 0.18 \\
Normal function & $5(25)$ & $35(32)$ & 0.61 \\
Aperistalsis & $6(30)$ & $40(37)$ & 0.62 \\
Anastomotic edema & $8(40)$ & $18(17)$ & $\mathbf{0 . 0 3}$ \\
CT Imaging & $22(50)$ & $157(84)$ & $\mathbf{0 . 0 0 0 1}$ \\
Fluid collection & $17(77)$ & $126(80)$ & 0.78 \\
Drainable collections & $5(23)$ & $94(60)$ & $\mathbf{0 . 0 0 1}$ \\
\hline
\end{tabular}

Table 4. Post TPN Outcomes

\begin{tabular}{lccc}
\hline \multicolumn{1}{c}{$\begin{array}{c}\text { Postoperative } \\
\text { Measure }\end{array}$} & $\begin{array}{c}\text { TPN } \leq 3 \text { Days } \\
n=44(\%)\end{array}$ & $\begin{array}{c}\text { TPN }>3 \text { Days } \\
n=188(\%)\end{array}$ & $p$-value \\
\hline CLABSI & $0(0)$ & $6(3)$ & 0.60 \\
Total Days on TPN & $2(1-3)$ & $12(4-115)$ & $\mathbf{0 . 0 0 0 1}$ \\
LOS (days) & $8.5(6-16)$ & $14(3-92)$ & $\mathbf{0 . 0 0 0 5}$ \\
Readmission & $16(36)$ & $66(35)$ & 0.86 \\
30-Day Mortality & $3(7)$ & $5(3)$ & 0.18 \\
\hline
\end{tabular}

\section{RESULTS}

Table 5. Multivariate Logistic Regression Analysis of Factors Affecting Length of TPN Use

\begin{tabular}{lccc}
\hline \multicolumn{1}{c}{ Risk Factor } & Odds Ratio & $95 \%$ CI & $p$-value \\
\hline Diabetes & 0.49 & $0.24-1.03$ & 0.060 \\
DGE & 1.22 & $0.57-2.60$ & 0.105 \\
Pancreatic Fistula & 2.57 & $1.03-6.41$ & $\mathbf{0 . 0 4 3}$ \\
Deep Surgical Site Infection & 3.09 & $1.16-5.06$ & $\mathbf{0 . 0 1 8}$ \\
\hline
\end{tabular}

\section{KEY FINDINGS}

$>$ The most common indications for TPN were delayed gastric emptying (DGE, $\mathrm{n}=171$, $73.7 \%$ ), pancreatic fistula ( $\mathrm{PF}, \mathrm{n}=102,44 \%$ ), and generalized malnutrition $(\mathrm{n}=25,10.8 \%)$.

$>$ The median day of TPN initiation was POD 4 (range: minus 31 to 22 ), with a median usage of nine days (range: 1 to 115).

$>$ Forty-four (19\%) patients received shortcourse TPN ( $\leq 3$ days), primarily those diagnosed with isolated Grade A DGE without associated complications ( $\mathrm{p}=0.0003$ ).

$>$ Multivariate analysis suggests the presence of deep surgical site infection (OR 3.09, [1.16 - 5.06], $\mathrm{p}=0.018$ ) or PF (OR 2.57, [1.03 6.41], $\mathrm{p}=0.043)$ at the time of DGE presentation as predictive of long-term TPN requirement.

\section{CONCLUSIONS}

$>$ TPN is safe and effective in patients following PD.

$>$ Avoid TPN in patients with isolated DGE secondary to anastomotic edema.

$>$ Use TPN in patients with DGE and another serious complication such as deep surgical site infection or PF. 